\title{
Hemoglobin S Measurement
}

National Cancer Institute

\section{Source}

National Cancer Institute. Hemoglobin S Measurement. NCI Thesaurus. Code C122123.

The determination of the amount of hemoglobin S present in a sample. 